\title{
É NECESSÁRIO O ESTUDO DO CÓLON NO FECHAMENTO DE COLOSTOMIAS?
}

\author{
HENRIQUE FRANCISCO DE SOUZA E SOUZA ${ }^{1}$, HERNÁN AUGUSTO CENTURIÓN SOBRAL ${ }^{1}$, ENZO MARTINS \\ TAGLIETTI $^{1}$, ELISÂNGELA PLAZAS MONTEIRO ${ }^{1}$, MARÍLIA REZENDE VON SONNLEITHNER GAMA ${ }^{1}$, \\ GALDINO JOSÉ SITONIO FORMIGA ${ }^{1}$
}

${ }^{1}$ Serviço de Coloproctologia do Hospital Heliópolis, São Paulo-SP, Brasil

SOUZA HFS, SOBRAL HAC, TAGLIETTI EM, MONTEIRO EP, GAMA MRVS, FORMIGA GJS. É Necessário o Estudo do Cólon no Fechamento de Colostomias? Rev bras Coloproct, 2006;26(2): 118-122.

RESUMO: O estudo pré-operatório do cólon para fechamento de colostomias em alça devido a trauma ainda é controverso. A pesquisa de alterações anatômicas pós-traumáticas do cólon entra em conflito com os custos, o desconforto e a morbidade dos exames. Objetivo: avaliar o fechamento de colostomia em alça, sem estudo pré operatório do cólon Método: foram analisados 51 pacientes do sexo masculino, no período de janeiro a junho de 2005, portadores de colostomia em alça confeccionada após traumatismo. Todos foram submetidos a fechamento de colostomia sem estudo do cólon, seja por colonoscopia ou enema opaco. Resultados: a média de idade foi de 26,5 anos. O tempo de permanência da colostomia foi, em média, 42,2 meses, sendo o flanco esquerdo a localização mais comum $(66,7 \%)$. Houve 16 casos $(31,4 \%)$ de hérnia para-colostômica e 14 casos $(27,5 \%)$ de procidência de colostomia. A morbidade foi de $13,7 \%$ e mortalidade ausente. As complicações mais freqüentes foram hematoma $(\mathbf{7 , 8 \%})$ e infecção $(3,9 \%)$ de ferida operatória e um caso de deiscência de anastomose (1,9\%). Conclusão: não é necessário o estudo do cólon pré-operatório de forma rotineira no paciente portador de colostomia em alça, após lesão traumática.

Descritores: Fechamento de colostomia, colostomia em alça, enema opaco, colonoscopia.

\section{INTRODUÇÃO}

Desde a Segunda Guerra Mundial, as colostomias temporárias vêm sendo utilizadas de forma rotineira como tratamento para ferimentos traumáticos colorretais ${ }^{1}$.
O fechamento de colostomia em alça é geralmente visto pela maioria dos cirurgiões como procedimento de fácil execução, entretanto, a literatura relata taxas de morbidade que variam de 10 a $49 \%{ }^{2,3}$.

O estudo pré-operatório do cólon, radiológico ou endoscópico, ainda é controverso, porém

Trabalho realizado no Serviço de Coloproctologia do Hospital Heliópolis, São Paulo-SP, Brasil

Recebido em 23/12/2005

Aceito para publicação em 13/02/2006 
and

publicações recentes vêm questionando a real necessidade destes exames ${ }^{4,5}$.

O objetivo deste trabalho é avaliar fechamento de colostomia em alça pós lesão traumática sem o estudo pré-operatório do cólon .

\section{PACIENTES E MÉTODO}

Foi realizado estudo prospectivo de 51 pacientes do sexo masculino procedentes do Sistema Penitenciário do Estado de São Paulo e portadores de colostomia em alça por ferimento traumático colorretal, sendo submetidos ao fechamento de colostomia no Serviço de Coloproctologia do Hospital Heliópolis, no período de janeiro a junho de 2005.

Procedeu-se ao exame clínico admissional de rotina, que consistia em avaliação da ostomia e exame proctológico com inspeção, toque retal, anuscopia e retoscopia. Hemograma, coagulograma e radiografia simples do tórax foram solicitados em todos os casos e demais exames complementares, nos casos em que eram pertinentes. Os pacientes não realizaram estudo préoperatório do cólon por enema opaco ou colonoscopia.

Todos os pacientes realizaram preparo do cólon via anterógrada e retrógrada. Foi administrado 1000 $\mathrm{ml}$ de solução de manitol $10 \%$ na véspera da cirurgia. $\mathrm{O}$ segmento excluso foi irrigado com $1000 \mathrm{ml} \mathrm{de}$ solução glicerinada a $12 \%$, quer por via retal ou via colostomia, o que nos dava noção da perviedade intestinal.
A anestesia utilizada foi geral e todos os pacientes receberam antibioticoprofilaxia com $500 \mathrm{mg}$ de metronidazol, $1 \mathrm{~g}$ de amicacina e $1 \mathrm{~g}$ de ampicilina, 30 minutos antes da indução anestésica.

As variáveis estudadas foram idade, tipo do trauma colorretal, local do estoma, tempo de permanência e avaliação ectoscópica da ostomia, técnica cirúrgica e evolução pós-operatória.

\section{RESULTADOS}

A média de idade foi de 26,5 anos, variando de 17 a 42 anos. O trauma que levou a confecção da ostomia foi ferimento por arma de fogo em 49 pacientes $(96 \%)$.

A localização mais freqüente da ostomia foi o flanco esquerdo em 34 pacientes $(66,6 \%)$, seguido pelo hipocôndrio direito $(15,6 \%)$, flanco direito e fossa ilíaca direita $(7,8 \%)$ e hipocôndrio esquerdo $(1,9 \%)$. (Figura-1).

O tempo médio de permanência da colostomia foi de 42,2 meses, variando de 2 meses a 10 anos. Aproximadamente $50 \%$ dos pacientes tinham a ostomia há mais de três anos. (Figura-2)

As complicações tardias da confecção da ostomia mais prevalentes foram procidência e hérnia paracolostômica, em $12 \%$ e $16 \%$ dos casos, respectivamente, estando associadas em $16 \%$.

O fechamento da colostomia foi feito com anastomose em plano único extra-mucoso com pontos

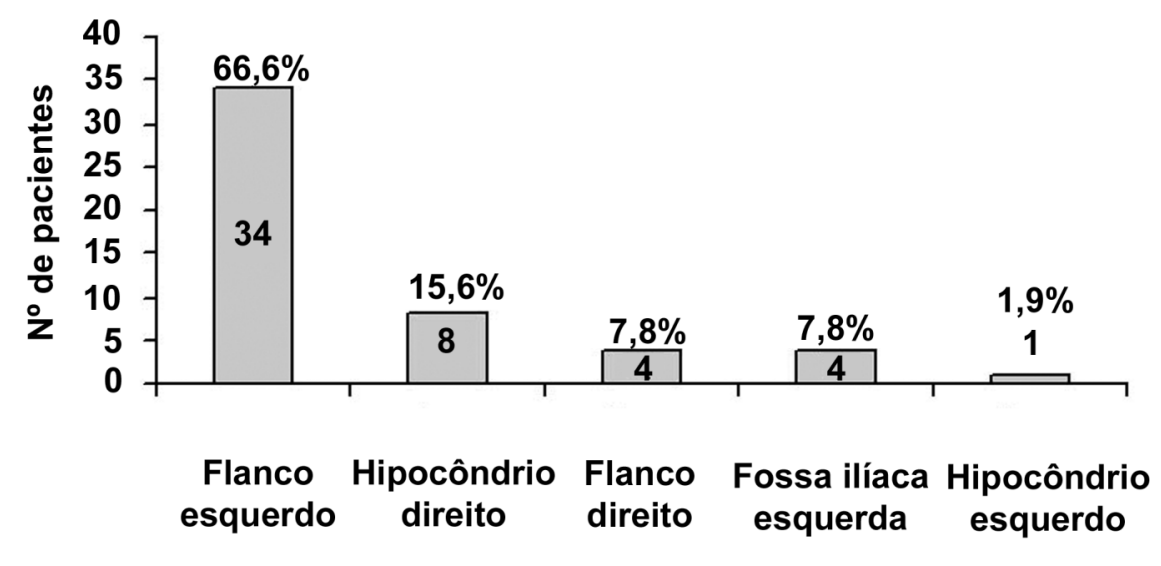

Local da ostomia

Figura 1 - Localização da ostomia na parede abdominal. 


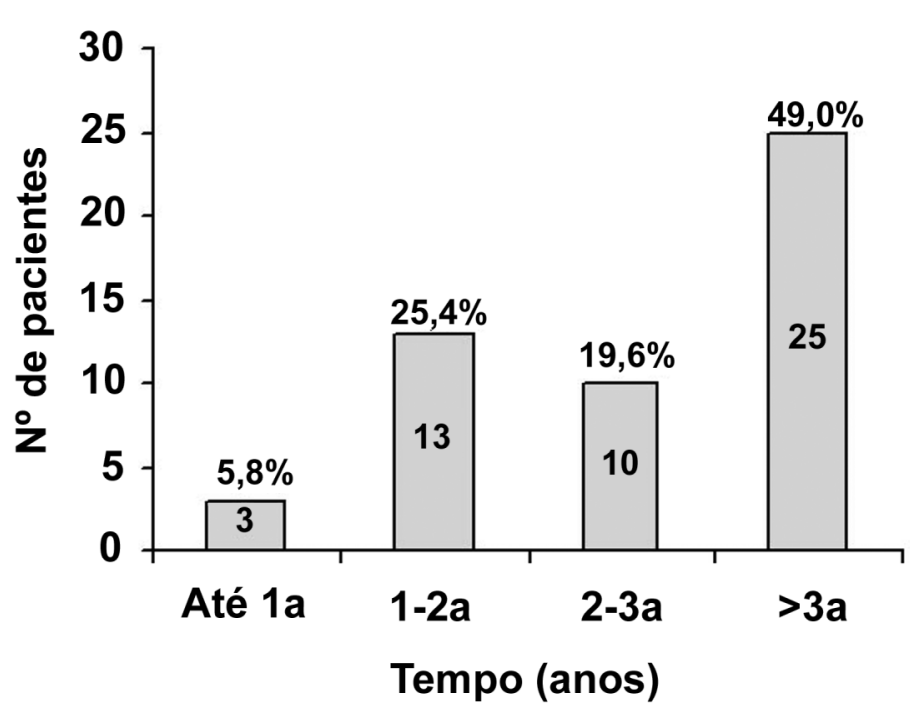

Figura 2 - Tempo de permanência da ostomia.

contínuos ou separados de fio inabsorvível (nylon, polipropileno ou algodão 3-0) ou em dois planos, sendo o primeiro muco-mucoso contínuo com fio absorvível (poligalactina 4-0) e o segundo plano conforme descrito anteriormente. Em 8 pacientes $(16 \%)$, houve a necessidade de realizar ressecção de pequeno segmento intestinal, não mais que $5 \mathrm{~cm}$, devido à lesão inadvertida durante a dissecção por intensa fibrose peri-colostômica e pontos de fixação aponeuróticos ou por desproporção das bocas a serem anastomosadas.
Setenta e seis por cento dos pacientes receberam dieta no primeiro dia de pós-operatório, seguido de $16 \%$ no segundo e $8 \%$ no terceiro. A dieta era introduzida na vigência de ruídos hidroaéreos propulsivos, ausência de vômitos e presença de eliminação de gases ou fezes.

Houve sete complicações imediatas, totalizando $13,7 \%$ da amostra, sendo quatro hematomas, duas infecções de ferida operatória e uma deiscência de anastomose, que foi abordada cirurgicamente. Não houve mortalidade. (Figura-3)

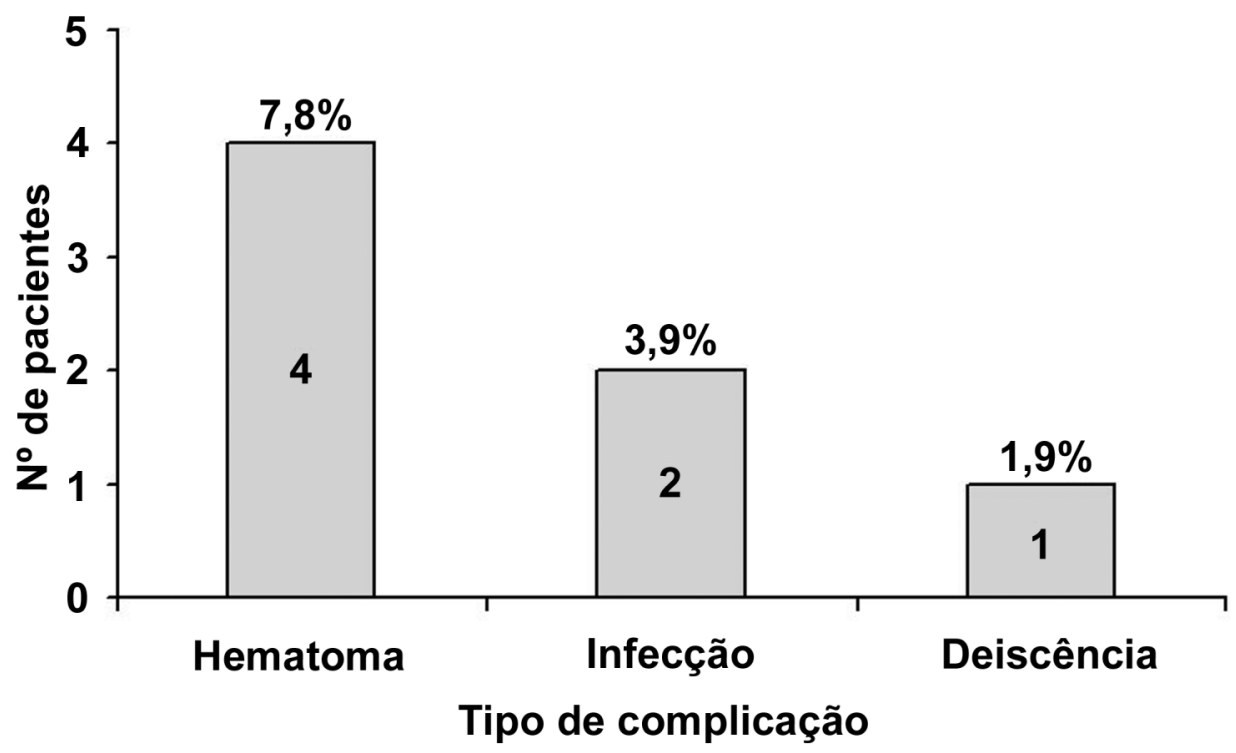

Figura 3 - Distribuição das complicações. 
A alta hospitalar foi concedida após boa aceitação dietética e funcionamento intestinal adequado. Desta forma, $84 \%$ dos pacientes receberam alta entre o $3^{\circ}$ e $5^{\circ}$ pós-operatório (PO) e apenas $16 \%$ após o $5^{\circ} \mathrm{PO}$.

\section{DISCUSSÃO}

A Segunda Guerra Mundial foi um marco histórico para o tratamento dos ferimentos traumáticos colorretais, quando se popularizou a utilização das colostomias ${ }^{1}$.

A exteriorização do cólon, apesar de comum na urgência, acaba por gerar um novo procedimento cirúrgico para o restabelecimento do trânsito intestinal. Apesar de muitos cirurgiões considerarem que as cirurgias para fechamento de colostomia são procedimentos simples, as taxas de complicação variam de 10 a $49 \%{ }^{2,3}$.

Outro aspecto controverso é a necessidade do estudo intestinal radiológico ou endoscópico préoperatório, sobretudo do segmento excluso. A utilização destes exames tem sido recomendada para certificar-se da integridade intestinal e avaliar a existência de alguma doença concomitante ${ }^{6-9}$. No entanto, enema opaco e colonoscopia, apesar da baixa morbidade, ocasionam aumento dos custos, desconforto para o paciente e atraso para o fechamento da colostomia. Além disso, tais exames estão normais na sua maioria, não alterando a conduta terapêutica. Deste modo, vários autores têm sugerido a não realização destes exames de forma rotineira $2,4,6,10,11$

Maximiano e cols, em 1995, após estudarem 89 pacientes, encontraram anormalidades no enema opaco pré-operatório em apenas 4,5\% dos casos. Estes pacientes apresentavam ferimentos complexos ou algum tipo de sintoma que sugeririam tais alterações ${ }^{11}$.
Outro aspecto relevante quanto à realização rotineira do estudo pré-operatório do cólon é o fato de a amostra estudada ser jovem, com média de idade de 26,5 anos, onde a possibilidade de encontrar doença colônica associada é baixa ${ }^{5}$.

O tempo de permanência da ostomia foi, em média, 42,2 meses, contrapondo ao da literatura consultada que variou de 5,7 a 9,8 meses ${ }^{4,11}$. Justificase o tempo prolongado de permanência da ostomia, por se tratar de pacientes oriundos do sistema penitenciário.

Como os pacientes participantes não foram submetidos a nenhum estudo pré-operatório do cólon, a realização de preparo retrógrado com solução glicerinada a $12 \%$ nos dava noção da perviedade intestinal.

A taxa de morbidade deste estudo foi de 13,7\%, sendo, na sua maioria, complicações de menor gravidade, como hematoma e infecção de ferida operatória, semelhante à de autores que utilizaram estudo do cólon, como Pokorny e Madiba, com morbidade de 15 e $14,5 \%$, respectivamente ${ }^{6,12}$.

Apesar de a idade do paciente, o motivo para confecção da colostomia, o local e o tempo de permanência do ostoma, bem como a utilização de drenos serem considerados fatores relacionados com a morbidade operatória, o único fator que reduz o índice de complicações pós-operatórias é a antibiótico-profilaxia ${ }^{5}$. O nosso único caso de deiscência de anastomose foi abordado cirurgicamente, sendo realizada nova colostomia derivativa, visto que a anastomose já se encontrava integrada e bloqueada.

Concluímos que o estudo pré-operatório do cólon possívelmente não é necessário, de forma rotineira, no paciente portador de colostomia em alça, após lesão traumática colorretal.

SUMMARY: Colorectal study for loop colostomy closure after traumatic injuries has been considered unnecessary, because of the cost, the patient's discomfort and the exams morbidity. Objective: to evaluate the real need of colorectal study preceding colostomy closure in trauma patients. Methods: fifty-one male patients were submitted to colostomy closure between January to June 2005. None of them were submitted to colorectal study before the surgery, neither barium enema nor colonoscopy. Results: the mean age was 26.5 years and the mean interval between colostomy creation and closure was $\mathbf{4 2 . 2}$ months. The left flank was the most common colostomy location $(66.6 \%)$. The morbidity rate was $13.7 \%$, with no mortality. Conclusion: routine colorectal study preceding colostomy closure is not necessary after colorectal injuries.

Key words: Colostomy closure, loop colostomy, barium enema, colonoscopy. 


\section{REFERÊNCIAS BIBLIOGRÁFICAS}

1. Garber HI; Morris DM; Eisenstat TE; Coker DD; Annous MO. Factors influencing the morbidity of colostomy closure. Dis Colon Rectum 1982;25(5):464-70.

2. Garnjobst W; Leaverton GH; Sullivan ES. Safety of colostomy closure. Am J Surg 1978;136(1):85-9.

3. Yakimets WW. Complications of closure of loop colostomy. Can J Surg 1975;18(4):366-70.

4. Sola JE; Buchman TG; Bender JS. Limited role of barium enema examination preceding colostomy closure in trauma patients. J Trauma 1994;36(2):245-6.

5. Demetriades D; Pezikis A; Melissas J; Parekh D; Pickles G. Factors influencing the morbidity of colostomy closure. Am J Surg 1988;155(4):594-6.

6. Madiba TE; Mahomva O; Haffejee AA; Nene B. Radio-contrast imaging of the rectum prior to colostomy closure for rectal trauma - is routine use still justified? S Afr J Surg 2000;38(1):17-8.

7. Atweh NA; Vieux EE; Ivatury R; Scalea TM; Duncan AO; Gordon $\mathrm{J}$ et al. Indications for barium enema preceding colostomy closure in trauma patients. J Trauma 1989;29(12):1641-2.
8. Thal ER; Yeary EC. Morbidity of colostomy closure following colon trauma. J Trauma 1980;20(4):287-91.

9. Parks SE; Hastings PR. Complications of colostomy closure. Am J Surg 1985;149(5):672-5.

10. Swenson K; Stamos M; Klein S. The role of barium enema in colostomy closure in trauma patients. Am Surg 1997;63(10):893-5.

11. Maximiano LF; Moron RA; Soh SW; Alves Jr A; Pires PWA; Bevilacqua RG; Biroloni D. Análise da necessidade do enema opaco como exame pré-operatório em fechamento de colostomias. Rev Col Bras Cir;1995;22(2):70-2.

12. Pokorny RM; Heniford T; Allen JW; Tuckson WB; Galandiuk S. Limited utility of preoperative studies in preparation for colostomy closure. Am Surg 1999;65(4):338-40.

\section{Endereço para correspondência:}

GALDINO JOSÉ SITONIO FORMIGA

Hospital Heliópolis - Serviço de Coloproctologia

Rua Cônego Xavier, 276 - Vila Heliópolis

04231-030 - São Paulo-SP

Tel: (11) 274-7600 (Ramal 244) 